\title{
Real-Time Data Assimilation for Operational Ensemble Streamflow Forecasting
}

\author{
JASPER A. VRUGT \\ Earth and Environmental Sciences Division, Los Alamos National Laboratory, Los Alamos, New Mexico \\ Hoshin V. GuPTA \\ Department of Hydrology and Water Resources, The University of Arizona, Tucson, Arizona \\ BREANNDÁN Ó NuALLÁIN \\ Department of Computational Science, University of Amsterdam, Amsterdam, Netherlands \\ WiLlem BOUTEN \\ Department of Physical Geography and Soil Science, University of Amsterdam, Amsterdam, Netherlands
}

(Manuscript received 16 March 2005, in final form 15 September 2005)

\begin{abstract}
Operational flood forecasting requires that accurate estimates of the uncertainty associated with modelgenerated streamflow forecasts be provided along with the probable flow levels. This paper demonstrates a stochastic ensemble implementation of the Sacramento model used routinely by the National Weather Service for deterministic streamflow forecasting. The approach, the simultaneous optimization and data assimilation method (SODA), uses an ensemble Kalman filter (EnKF) for recursive state estimation allowing for treatment of streamflow data error, model structural error, and parameter uncertainty, while enabling implementation of the Sacramento model without major modification to its current structural form. Model parameters are estimated in batch using the shuffled complex evolution metropolis stochasticensemble optimization approach (SCEM-UA). The SODA approach was implemented using parallel computing to handle the increased computational requirements. Studies using data from the Leaf River, Mississippi, indicate that forecast performance improvements on the order of $30 \%$ to $50 \%$ can be realized even with a suboptimal implementation of the filter. Further, the SODA parameter estimates appear to be less biased, which may increase the prospects for finding useful regionalization relationships.
\end{abstract}

\section{Introduction and scope}

Since the early 1960s, considerable effort has been devoted to the development and application of models of the rainfall-runoff process. A class of these, often called "conceptual watershed models" represents the precipitation-soil moisture-streamflow water balance dynamics using heuristic and/or empirical relationships that represent a perceptual and conceptual hydrologic understanding of watershed behavior at aggregate scales (see, e.g., Kuczera 1997). Conceptual watershed

Corresponding author address: Jasper Vrugt, Earth and Environmental Science Division, Los Alamos National Laboratory, Los Alamos, NM 87544.

E-mail: vrugt@lanl.gov models used for operational streamflow forecasting typically have 10 or more parameters that specify the behavior of the transfer functions relating inputs to outputs via series and parallel pathways of interconnected conceptual water storages (state variables) representing soil moisture accumulations in the upper and lower soil zones. It is tacitly assumed that these conceptual storages correspond to "real" control volumes in physical space, even though the boundaries of these control volumes cannot generally be explicitly delineated.

The Sacramento Soil Moisture Accounting conceptual watershed model (SAC-SMA; Burnash et al. 1973) is used by the National Weather Service (NWS) for operational streamflow forecasting and flood warning throughout the United States. The model has 16 parameters whose values must be specified (Table 1). 
TABLE 1. Parameter and state variables in the SAC-SMA model.

\begin{tabular}{|c|c|c|c|c|c|}
\hline \multicolumn{3}{|c|}{ Capacity thresholds } & $\begin{array}{l}\text { Initial } \\
\text { ranges }\end{array}$ & $\begin{array}{l}\text { SCEM-UA } \\
\text { ranges }\end{array}$ & $\begin{array}{l}\text { SODA } \\
\text { ranges }\end{array}$ \\
\hline UZTWM & Upper-zone tension water maximum storage & $(\mathrm{mm})$ & $1.0-150.0$ & $90.2-91.1$ & $85.8-129.3$ \\
\hline UZFWM & Upper-zone free water maximum storage & $(\mathrm{mm})$ & $1.0-150.0$ & $16.3-16.8$ & $66.5-93.6$ \\
\hline LZTWM & Lower-zone tension water maximum storage & $(\mathrm{mm})$ & $1.0-500.0$ & $267.6-270.2$ & $121.2-155.3$ \\
\hline LZFPM & Lower-zone free water primary maximum storage & $(\mathrm{mm})$ & $1.0-1000.0$ & $141.8-148.2$ & $384.6-730.4$ \\
\hline LZFSM & Lower-zone free water supplemental maximum storage & $(\mathrm{mm})$ & $1.0-1000.0$ & $51.0-53.9$ & $180.0-557.4$ \\
\hline \multicolumn{6}{|c|}{$\begin{array}{l}\text { Additional impervious area } \\
\text { Recession parameters }\end{array}$} \\
\hline UZK & Upper-zone free water lateral depletion rate & $\left(\right.$ day $\left.^{-1}\right)$ & $0.1-0.5$ & $0.50-0.50$ & $0.32-0.46$ \\
\hline LZPK & Lower-zone primary free water depletion rate & $\left(\right.$ day $\left.^{-1}\right)$ & $0.0001-0.025$ & $0.001-0.001$ & $0.0006-0.004$ \\
\hline \multicolumn{6}{|c|}{ Percolation and other } \\
\hline ZPERC & Maximum percolation rate & $(-)$ & $1.0-250.0$ & $248.9-250.0$ & $4.5-94.8$ \\
\hline REXP & Exponent of the percolation equation & $(-)$ & $1.0-5.0$ & $2.6-2.7$ & $1.2-2.1$ \\
\hline PCTIM & Impervious fraction of the watershed area & $(-)$ & $0.0-0.1$ & $0.007-0.007$ & $0.04-0.05$ \\
\hline PFREE & $\begin{array}{l}\text { Fraction percolating from upper- to lower-zone free } \\
\text { water storage }\end{array}$ & $(-)$ & $0.0-0.6$ & $0.13-0.13$ & $0.37-0.44$ \\
\hline \multicolumn{6}{|c|}{ Not optimized } \\
\hline RIVA & Riparian vegetation area & $(-)$ & 0.0 & & \\
\hline SIDE & Ratio of deep recharge to channel base flow & $(-)$ & 0.0 & & \\
\hline RSERV & $\begin{array}{l}\text { Fraction of lower zone free water not transferable to } \\
\text { tension water }\end{array}$ & $(-)$ & 0.3 & & \\
\hline States & Description & & & & \\
\hline UZTWC & Upper-zone tension water storage content & $(\mathrm{mm})$ & & & \\
\hline UZFWC & Upper-zone free water storage content & $(\mathrm{mm})$ & & & \\
\hline LZTWC & Lower-zone tension water storage content & $(\mathrm{mm})$ & & & \\
\hline LZFPC & Lower-zone free primary water storage content & $(\mathrm{mm})$ & & & \\
\hline LZFSC & Lower-zone free secondary water storage content & $(\mathrm{mm})$ & & & \\
\hline ADIMC & Additional impervious area content & $(\mathrm{mm})$ & & & \\
\hline
\end{tabular}

While the values of some of these parameters can be estimated directly from knowledge of physical watershed characteristics, most represent "effective" watershed properties that cannot, in practice, be measured via direct observation in the field. It is common practice, therefore to estimate values for the model parameters by calibrating the performance of the model against a historically observed streamflow hydrograph, using either a "manual expert" trial-and-error approach or an automated search algorithm (e.g., Gupta et al. 1998; Boyle et al. 2000; Madsen 2000). Note that parameters estimated in this manner are spatially and temporally lumped (i.e., effective) representations of heterogeneous watershed properties.

While considerable progress has been made in the development and application of automated procedures for watershed model calibration, such methods have received criticism for their lack of rigor in treating various sources of uncertainty (e.g., Beven and Binley 1992; Thiemann et al. 2001; Vrugt et al. 2005). In particular, most procedures explicitly account only for uncertainties in the streamflow measurement data (e.g., Sorooshian and Dracup 1980; Sorooshian et al. 1993) and in the parameter estimates (e.g., Kuczera 1983a,b;
Beven and Binley 1992; Gupta et al. 1998; Thiemann et al. 2001; Vrugt et al. 2003b, 2006a). Most methods use batch processing of the data to search for parameter estimates that minimize a likelihood measure of the overall (statistical) variance of the model residuals. Uncertainties that stem from errors associated with the measured system inputs (rainfall and potential evapotranspiration), initialization and propagation of state variables (moisture storages), and model structural errors arising from inadequate representation of physical processes (including the aggregation of spatially distributed processes) have not been adequately handled in an explicit manner.

Over the past decade, interesting methods for addressing such problems have appeared in the literature, with attention given to the problem of estimating reasonable uncertainty bounds on the model predictions. Bayesian, pseudo-Bayesian, set-theoretic, multiplecriteria, and recursive model identification strategies that estimate model parameter and streamflow prediction uncertainties can provide forecasts of the "most probable" streamflow value along with estimates of the ranges of possible outcomes (e.g., Kuczera 1983a,b; Keesman 1990; van Straten and Keesman 1991; Beven 
and Binley 1992; Klepper et al. 1991; Freer et al. 1996; Gupta et al. 1998; Thiemann et al. 2001; Young 2001; Vrugt et al. 2003b, 2006a). Such methods typically summarize the model uncertainty primarily in terms of uncertainty in the parameter estimates.

Since the early 1980s, state-space filtering methods have been proposed as having the potential for explicitly handling uncertainties in hydrological models. In contrast to classical model calibration strategies, statespace filtering methods continuously update the states in the model when new measurements become available, to improve the model forecast and estimate forecast accuracy. Variations of the Kalman filter approach (Kalman 1960) have been applied to real-time streamflow forecasting by Todini et al. (1976), Kitanidis and Bras (1980a,b), Bras and Restrepo-Posada (1980), Bras and Rodriguez-Iturbe (1985), Wood and O'Connell (1985), Awwad and Valdés (1992), Awwad et al. (1994), and Young (2002), among others. Of particular relevance to this paper is the pioneering work of Kitanidis and Bras (1980a,b) in which an extended Kalman filter (EKF) was implemented using a state-space reformulation of the nonlinear SAC-SMA model to track probabilistic estimates of the state variables and to detect impulse-type rainfall errors. However, the recursive KF approach did not achieve practical operational status for a variety of practical reasons, an important one being that the implementation requires the highly nonlinear original model to be rendered into a continuously differentiable state-space form, involving various modifications and/or approximations (Kitanidis and Bras 1980a,b; Georgakakos et al. 1988; Georgakakos and Sperflage 1995; Refsgaard 1998; Seo et al. 2003). Besides the fact that the input-state-output behavior of the reformulated state-space model can deviate significantly from the original model- the EKF is notoriously unstable if the model nonlinearities are strong (Evensen 1992; Miller et al. 1994)—-the model reformulation and the computation of model derivative equations can require a considerable amount of experience and expertise (Seo et al. 2003). Further, the computational costs of state-space filtering methods (e.g., KF and EKF) are significant (especially for high-dimensional state-vector problems, such as spatially distributed models) and have, so far, restricted their widespread implementation for operational use.

Other data assimilation approaches have been recently explored. Seo et al. (2003) investigated variational assimilation (VAR) as a tool for assimilating climatological estimates of potential evaporation and real-time observations of streamflow and precipitation to improve SAC-SMA model streamflow forecasts. The VAR method has achieved widespread application to hydrometeorological and oceanographic models and does not depend on a state-space model formulation, but does require availability of an adjoint code that can be difficult to derive. Hydrological investigations of VAR include the work of Reichle et al. (2001) for estimating spatial soil-moisture distributions by assimilation of remotely sensed microwave brightness temperatures into a land surface model. Madsen and Skotner (2005) investigated the suboptimal steady Kalman filter approximation of Cañizares et al. (2001) for adaptive state updating using the Mike-11 model and reported that the reduced computational costs make the method better suited than the full Kalman filter for operational use.

In the past few years, ensemble forecasting techniques based on sequential data assimilation (SDA) methods have become increasingly popular due to their potential ability to explicitly handle the various sources of uncertainty in operational hydrological models. Early techniques reported in the literature include the dynamic identifiability analysis approach for recursive identifiability and detection of time variation in model parameters (DYNIA; Wagener et al. 2003), the parameter identification method based on localization of information (PIMLI; Vrugt et al. 2002), and the Bayesian recursive estimation approach (BaRE; Thiemann et al. 2001; Misirli 2003; Gupta et al. 2003). Most recently, techniques based on the ensemble Kalman filter (EnKF; Evensen 1994) have been suggested as having the power and flexibility required for data assimilation using conceptual watershed models. Vrugt et al. (2005) presented the simultaneous optimization and data assimilation method (SODA), which uses EnKF to recursively update model states while estimating time-invariant values for the model parameters using the shuffled complex evolution metropolis stochastic-ensemble optimization approach (SCEM-UA; Vrugt et al. 2003b). A novel feature of SODA is its explicit treatment of errors due to parameter uncertainty, state variables' uncertainty, model structural error, and streamflow measurement error. Moradkhani et al. (2005a,b) presented two different dual state-parameter estimation (DSPE) methods based on EnKF and sequential Monte Carlo techniques [SMC; also known as particle filtering (e.g., Gordon et al. 1993; Arulampalam et al. 2002)], respectively, for simultaneous recursive time-variable estimation of model states and parameters. Both EnKF and SMC allow accurate tracking of second-order moments of the probability distribution functions for models having nonlinear dynamics, but SMC can also track higher moments and therefore incurs increased computational costs. The EnKF achieves better computational efficiency by using linear rules for state updating but 


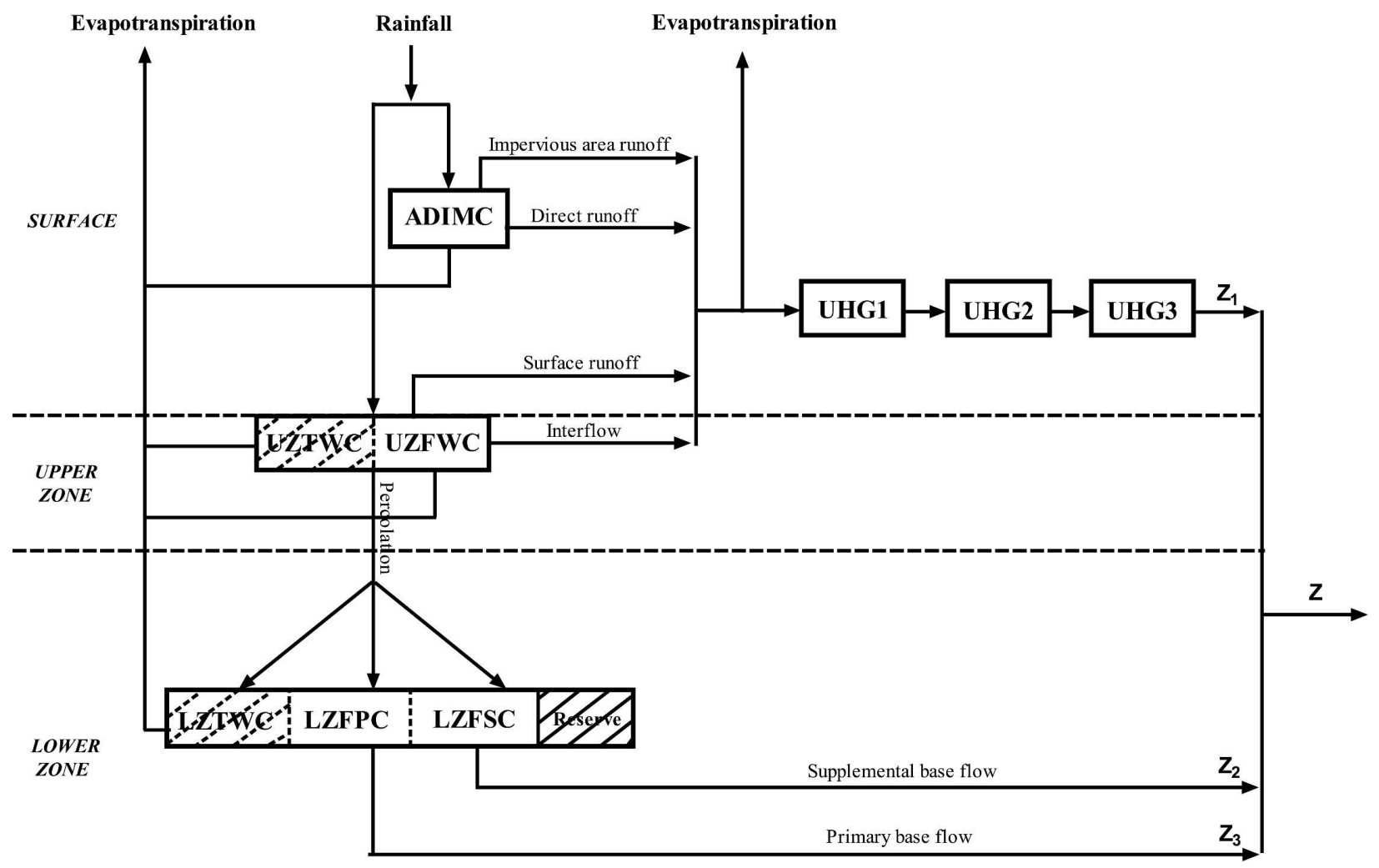

FIG. 1. Schematic representation of the SAC-SMA model as implemented in this study. The rectangles refer to the various model states, while arrows indicate fluxes between compartments; $Z_{1}, Z_{2}$, and $Z_{3}$ refer to the three main channel components, which sum together to become the streamflow $Z$ at the watershed outlet.

may give suboptimal performance in the presence of strong model nonlinearities.

The objective of this paper is to investigate the applicability and advantages of using SODA for joint parameter-state estimation and ensemble streamflow forecasting using the operational SAC-SMA conceptual watershed model. Our motivation is that the SODA method has the potential for ready assimilation into NWS operational forecasting procedures, thereby providing stochastic streamflow forecasts without requiring significant modifications to operational model codes or software. The paper is organized as follows. Section 2 presents a brief description of the SAC-SMA model. Sections 3 and 4 discuss the rationale and architecture of SODA and its implementation using parallel computing. Sections 5 and 6 present the experimental design and data used in this study and discuss the findings and results. In particular, we compare the performance of the stochastic SODA method with the conventional deterministic approach in current operational usage. Finally, section 7 summarizes the results and conclusions and explores implications for future work.

\section{Sacramento Soil Moisture Accounting model}

The SAC-SMA model (Fig. 1) is a lumped conceptual watershed model consisting of six state variable reservoirs representing the accumulation of water in two soil zones (upper and lower), in the forms of both "tension" and "free" water (Burnash et al. 1973; Brazil and Hudlow 1981). The upper zone represents surface soil processes and interception storage, while the lower zone represents deeper soil processes and groundwater storage. Nonlinear equations relate the absolute and relative quantities of water within the state variable reservoirs and control the partitioning of precipitation into overland flow, infiltration to the upper zone, interflow, percolation to the lower zone, and fast and slow components of groundwater recession baseflow. Saturation excess overland flow occurs when rainfall exceeds the interflow and percolation capacities and the upper zone storage is full. Percolation from the upper to the lower layer is controlled by a complex nonlinear process dependent on the storages in both soil zones.

The model has 13 user-specifiable (and 3 fixed) parameters and an evapotranspiration demand curve (or 
adjustment curve). Inputs to the model include mean areal precipitation (MAP) and potential evapotranspiration (PET) while the outputs are estimated evapotranspiration and channel inflow. A unit hydrograph or kinematic wave routing model is commonly used to rout channel inflow downstream to the gauging point. In this work, we instead use a simple Nash cascade (NC) of three linear reservoirs to rout the upper zone (quick response) channel inflow $\left(Z_{1}\right)$ while the lower zone recession components $Z_{2}$ and $Z_{3}$ are passed directly to the gauging point. This configuration adds one parameter and three state variables to the model, but facilitates inclusion of the $\mathrm{NC}$ routing model parameter and states in the data assimilation process. Moreover, NC routing considerably improves computational time as it avoids the need for computationally expensive convolution. Our formulation of the model therefore has 14 time-invariant parameters and 9 time-varying state variables to be estimated.

The SAC-SMA model has been used extensively in previous studies to study automatic model calibration issues (e.g., Brazil and Hudlow 1981; Sorooshian and Gupta 1983; Gupta and Sorooshian 1985; Sorooshian et al. 1993; Gupta et al. 1998; Boyle et al. 2000, 2001; Hogue et al. 2000, 2005; Thiemann et al. 2001; Vrugt et al. 2003a, 2006a; among others) and to explore state estimation for improved streamflow forecasting (e.g., Kitanidis and Bras 1980a,b; Seo et al. 2003).

\section{Simultaneous optimization and data assimilation}

Conventional methods for automated calibration of nonlinear watershed models treat the underlying uncertainty in the input-state-output representation of the model as arising primarily from uncertainty in the parameter estimates and output measurements. That approach essentially neglects measurement errors associated with the system input (forcing), state variables, and model structure leading to model simulations and associated prediction uncertainty bounds that do not consistently and accurately simulate the observed uncertainty in system behavior (Vrugt et al. 2005). This is easily seen by examination of the model residuals, which typically exhibit considerable variation in bias (nonstationarity), variance (heteroscedasticity), and correlation structure under different hydrologic conditions. Several contributions to the hydrologic literature have brought into question the continued usefulness of the classical paradigm for estimating model parameters (see, e.g., Beven and Binley 1992; Gupta et al. 1998; Kavetski et al. 2003).

In a separate line of research considerable progress has been made in the development and application of
SDA techniques, which provide a general and explicit framework for dealing with input, output, and model structural uncertainties but assume the optimal model parameters to be known a priori. The latter assumption is particularly inappropriate for conceptual watershed models, in which the parameters represent lumped and aggregated processes in space and time that cannot be measured directly and are therefore poorly specified. It has been our experience that considerable uncertainty in the state values and associated model output prediction can arise due to uncertainty in the choice of parameter values.

Vrugt et al. (2005) recently proposed the combined use of parameter optimization and sequential data assimilation to facilitate improved treatment of input, output, parameter, and model structural errors in hydrologic modeling using an algorithm named SODA. The algorithm merges the strengths of the SCEM-UA stochastic parameter optimization method with the power and computational efficiency of EnKF to simultaneously estimate both state variables and parameters. For detailed descriptions of EnKF and SODA see Evensen (1994) and Vrugt et al. (2005), respectively. In brief, SODA implements an inner EnKF loop for recursive state estimation (conditioned on an assumed parameter set) within an outer stochastic global optimization loop for batch estimation of the posterior density of the parameters. The EnKF uses a Monte Carlobased randomly sampled ensemble of state trajectories to propagate and update approximate estimates of the mean and covariance of the uncertain state variables $\psi_{t}$ from one time step to the next. See Fig. 2 for a condensed outline of the method. A key point is that the EnKF propagates an ensemble of state-vector trajectories in parallel such that each trajectory represents one realization of generated model replicates. When an output measurement is available, each forecasted ensemble state vector $\boldsymbol{\psi}_{t}^{f}$ is updated to $\boldsymbol{\psi}_{t}^{u}$ by means of a linear updating rule (in a manner analogous to the Kalman filter):

$$
\boldsymbol{\psi}_{t}^{u}=\boldsymbol{\psi}_{t}^{f}+K_{t} \cdot\left[\tilde{z}_{t}-H\left(\boldsymbol{\psi}_{t}^{f}\right)\right],
$$

where $\tilde{z}_{t}$ denotes a streamflow observation from dataset $\tilde{Z}=\left\{\tilde{z}_{1}, \ldots, \tilde{z}_{n}\right\}, H(\cdot)$ represents the operator relating the model states to the model output, and the strength of the gain $K_{t}$ depends on the strength of the cross covariance between the state variables of interest and the model outputs for which measurements are available. The cross covariance is approximated using the information contained in the ensembles. It should be noted, therefore, that the EnKF updating strategy favors situations for which the state to output transition 


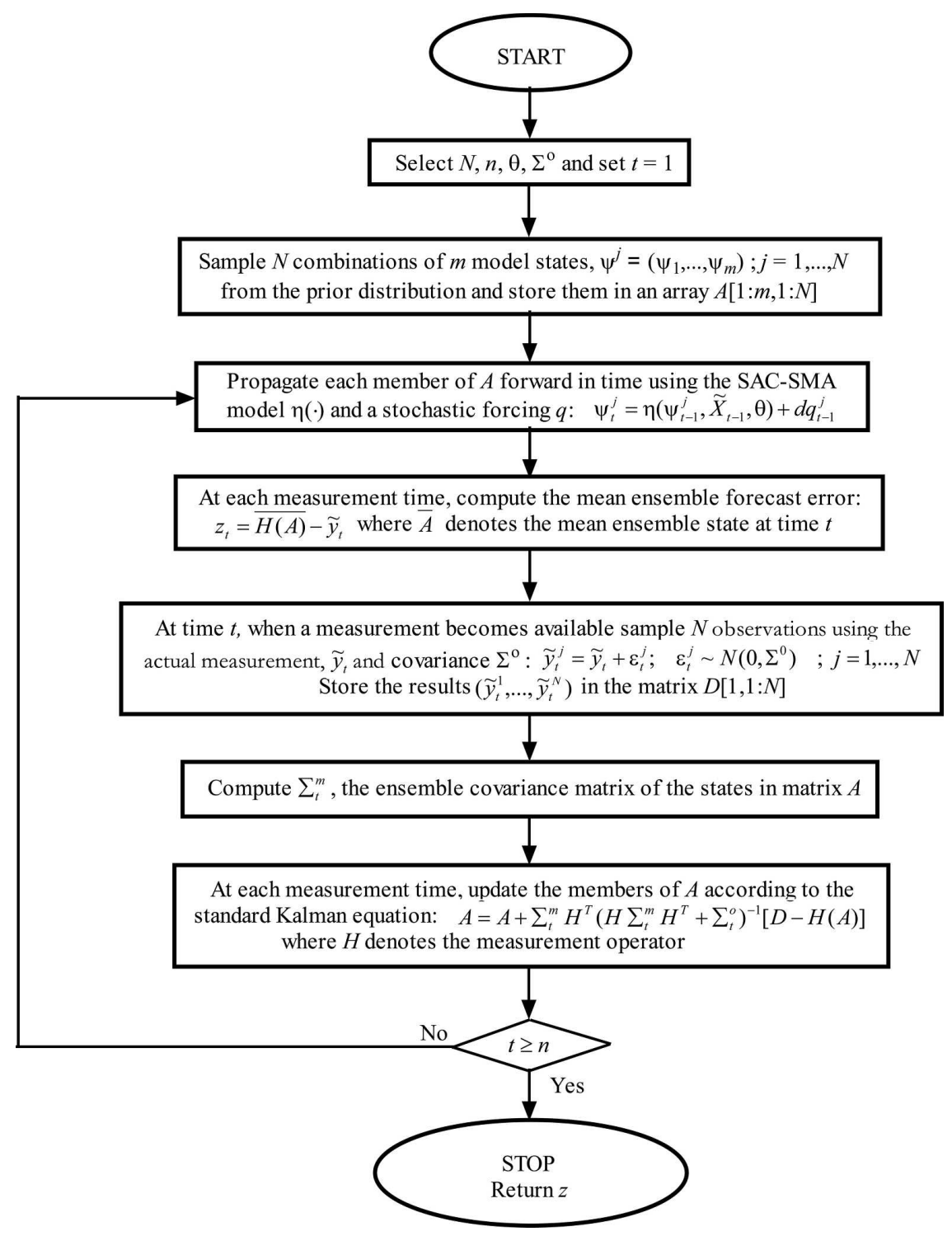

FIG. 2. Flowchart of the EnKF used in SODA to recursively estimate the state variables; $N$ denotes the ensemble size, $n$ the number of time steps, $\theta$ is the SCEM-UA generated parameter combination, and $\Sigma^{\circ}$ represents the error covariance of the observations.

equations are weakly nonlinear or almost linear over the range of conditions spanned by the ensembles, and for cases where the state-output relationships are strongly nonlinear, the EnKF may not be useable for state updating.

For parameter estimation, a Bayesian criterion is specified (Box and Tiao 1973) that measures the "closeness" between the (EnKF derived) mean ensemble model forecast and the corresponding measurement, resulting in the posterior density function:

$$
p(\theta \mid \tilde{Z}) \propto \sum_{t=1}^{n}\left[e_{t}(\theta)\right]^{-n},
$$

where $n$ denotes the total number of streamflow observations, and $e_{t}(\theta)$ represents the mean ensemble streamflow forecast error computed at each measurement time $t$ as

$$
e_{t}(\theta)=\tilde{z}_{t}-H\left[\boldsymbol{\psi}_{t}^{f}(\theta)\right]
$$

To generate parameter samples corresponding to the distribution indicated by the posterior parameter probability density function [Eq. (2)], we use an implementation of the general purpose SCEM-UA global optimization algorithm, which provides an efficient estimate of the posterior distribution and its mode (the 
most likely parameter set) within a single optimization run (Vrugt et al. 2003b). The algorithm, a modification of the successful SCE-UA global optimization method (Duan et al. 1992, 1994), belongs to the family of Markov chain Monte Carlo (MCMC) samplers, and generates multiple sequences of parameter sets $\left\{\theta^{(1)}, \theta^{(2)}, \ldots, \theta^{(\mathrm{k}+1)}\right\}$ that converge to the stationary posterior distribution. Studies using a parsimonious five-parameter conceptual watershed model have shown that SODA leads to improved estimates of parameter and model output prediction uncertainty (Vrugt et al. 2005).

\section{Implementation using parallel computing}

Implementation of the SODA methodology requires the EnKF solution of a sequential state estimation problem (over the calibration period) for each SCEMUA generated parameter set. Approximation of the stochastic evolution of the state error covariance matrix therefore involves large numbers of deterministic watershed model simulation runs. Fortunately, there has been considerable progress in the development of distributed computer systems using the power of multiple processors to efficiently solve large computational problems. In hydrology, many computational problems related to the flow and storage of water are admirably suited for implementation on distributed computer systems.

In this study, we implemented the SODA methodology using a local area multi-computer (LAM)/message passing interface (MPI) distributed computing interface for the Octave programming environment (Vrugt et al. 2006b). LAM/MPI is a high-quality open-source implementation of the message passing interface specification, including all of MPI-1.2 and much of MPI-2. Intended for production as well as research use, LAM/ MPI includes a rich set of features for parallel computing. GNU Octave (Eaton 1998, 2001) is a highlevel language intended primarily for numerical computation. It provides a convenient command line interface for numerical solution of linear and nonlinear problems, and for performing numerical experiments, using a language that is highly compatible with MATLAB. A detailed description and explanation of the software appear in Fernández et al. $(2003,2004)$.

Our parallel computing implementation of SODA incorporates some minor modifications to the sequential nature in which the samples are generated within each Markov chain of the SCEM-UA algorithm. A flowchart of our parallel implementation is given in Fig. 3. First, the evaluation of the fitness function for the individuals in the population is distributed over the slave proces- sors, thereby avoiding excessively long execution times on a single processor. Second, each slave computer (node) is set up to evolve a different sequence and complex in the SCEM-UA algorithm, as this step does not require information exchange and communication between different nodes. Each node might therefore be considered as a "particle" within the state space, evolving each of the SCEM-UA Markov chains to explore the global parameter space. The calculations reported in this paper were implemented using 25 Pentium IV 3.40-GHz processors of the LISA cluster belonging to the SARA parallel computing center (Netherlands). The CPU time required for joint stochastic calibration and ensemble state estimation of the SAC-SMA model using $8 \mathrm{yr}$ of daily streamflow data and 50000 SCEMUA generated parameters combinations was approximately $22 \mathrm{~h}$.

\section{Experimental design and data used}

The watershed used for this study is the Leaf River basin in Mississippi for which approximately $40 \mathrm{yr}$ of historical 6-hourly MAP and daily streamflow and PET data are available. Previous work has indicated that a calibration dataset of approximately 8 to $11 \mathrm{yr}$ of data, representing a range of hydrologic phenomena (wet, medium, and dry years), is desirable to achieve deterministic model calibrations that are consistent and generate good verification and forecasting performance (e.g., Yapo et al. 1996; Vrugt et al. 2006a). In this study we use 8 yr [water years (WY) 1953-60] for model calibration and further 28 yr (WY 1961-88) for evaluation of model forecast performance.

Two calibration cases are considered. In the first (benchmark) case, called "deterministic" in the text, we calibrate and evaluate the SAC-SMA model in the conventional manner, using SCEM-UA to estimate parameter uncertainty, without state-variable updating. The wording deterministic is used to reflect that we do not use an explicit (stochastic) model error, but instead assume that the SAC-SMA model is a correct representation of the underlying rainfall-runoff transformation. A standard maximum likelihood estimation criterion is used based on a heteroscedastic Gaussian error model for the streamflow measurements, with the measurement error variances specified explicitly as explained later in this section. The prediction performance of the calibrated deterministic model is then evaluated by projecting the estimated parameter uncertainty into the output space and comparing with the measured streamflow hydrograph.

In the second case, called "stochastic" in the text, we use the SODA data assimilation methodology to cali- 


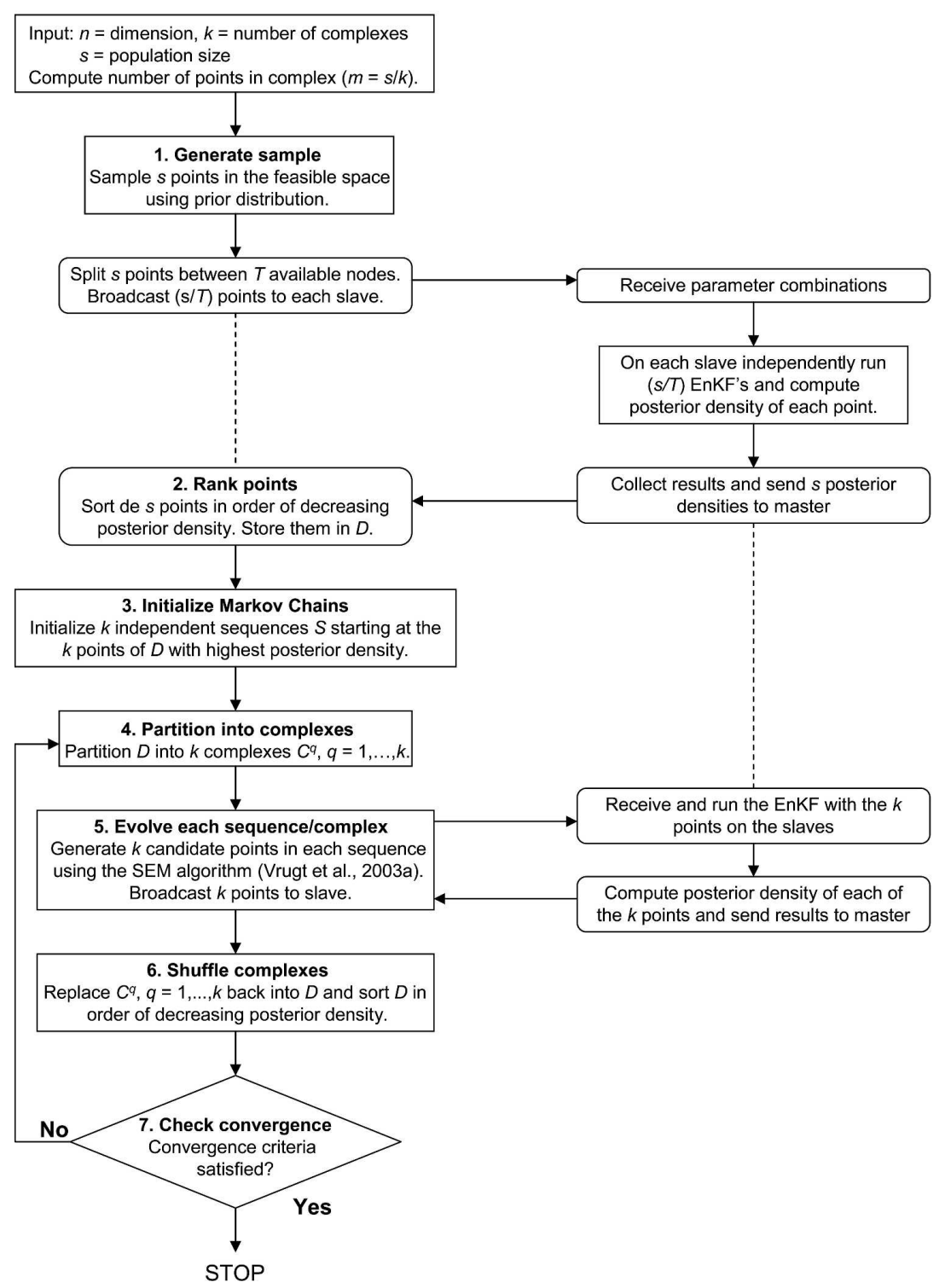

FIG. 3. Schematic overview of the parallel implementation of SODA. The master computer performs the various algorithmic steps in the SCEM-UA algorithm, while the slave computers solve the computationally expensive recursive state estimation problem using the EnKF.

brate the model parameters (in batch mode), using SCEM-UA to estimate parameter uncertainty, with (recursive mode) state-variable updating using an EnKF applied to each sampled parameter set. In this case, we evaluate the model performance under two conditions-first in simulation mode without recursive assimilation of streamflow measurement data, and then in online data assimilation mode using the EnKF to update the model states as streamflow observations become available. In both evaluation cases the parameters of the model are fixed to the constant values es- timated during calibration. The first of these two evaluation cases emulates the standard deterministic forecasting procedure except that streamflow uncertainty bounds based on parameter uncertainty are also provided, while the second evaluation case illustrates the performance achievable using recursive online data assimilation for state updating.

In each calibration case, proper specification of the likelihood function used to extract information from the streamflow data requires realistic estimates of the statistical properties of the streamflow measurement 
error. In the absence of compelling information describing the statistical properties of the measurement error, most previous studies reported in the literature have assumed that the measurement error can be described by a Gaussian distribution having zero mean and having a prespecified parametric functional form that relates the error variance to the magnitude of the associated flow value (e.g., Sorooshian and Dracup 1980; Thiemann et al. 2001; Vrugt et al. 2003b, 2006a; among others). The parameters of this variance function are then jointly estimated during calibration, along with the parameters of the model, from the properties of the model residual. However, because of input, state, and structural errors the statistics of the model residual will not necessarily behave in a fashion similar to the statistics of the streamflow measurement error. In this paper, we use an alternative nonparametric approach for estimating the properties of the measurement error in a data series (e.g., Rice 1984; Hall et al. 1990; Seifert et al. 1993; Dette et al. 1998). This method assumes that the measurement errors are random in nature, and involves taking the time difference of the original time series, $\tilde{z}_{t}$, and estimating the error deviation as

$$
\hat{\sigma}_{t}^{o}=\sqrt{\frac{1}{2(n-1)} \sum_{t=2}^{n}\left(\tilde{z}_{t}-\tilde{z}_{t-1}\right)^{2}},
$$

which assumes that the time sequence of flow values is sufficiently smooth, the sampling interval is sufficiently high compared to the temporal scale of streamflow dynamics, and that the variance of the measurement error is constant (homoscedastic). While the first two assumptions might be reasonable for daily streamflow time series, the assumption of homoscedasticity of the streamflow error terms is unrealistic. We therefore instead apply the nonparametric error deviation estimator "locally" to the time series using

$$
\hat{\sigma}_{t}^{o}=\sqrt{\left(\begin{array}{c}
2 u \\
u
\end{array}\right)^{-1}\left(\Delta^{u} \tilde{z}_{t}\right)^{2}}
$$

where $\Delta^{u}$ denotes the difference operator applied $u$ times. It can be readily verified that the estimator in Eq. (5) is insensitive to polynomial trends in the data up to order $u$. While more sophisticated higher-order differencing procedures have been proposed (Hall et al. 1990), our investigations with numerically generated streamflow data have shown that a choice of $u=3$ works well in practice (Vrugt et al. 2005). The optimal value of $u$ can be determined by evaluating Eq. (5) over a range of $u$ values and comparing the error estimates as a function of flow level with the error functions used to perturb the synthetic streamflow observations. Fig-

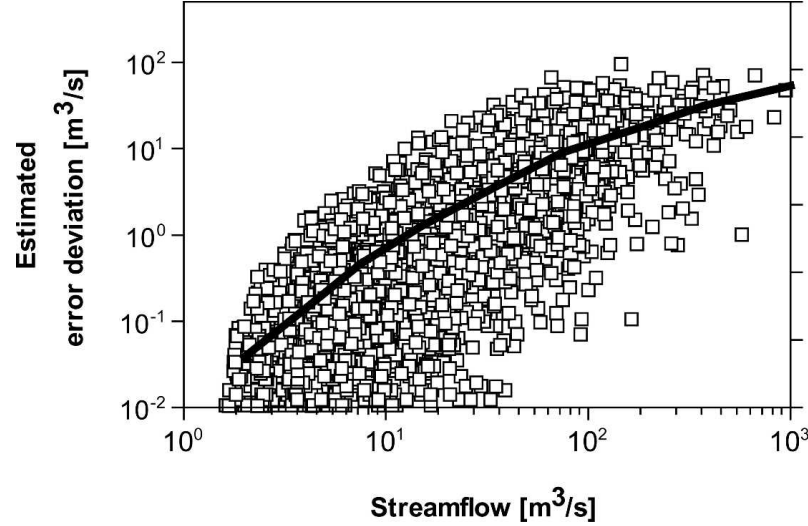

FIG. 4. Scatterplot (square symbols) of the error deviation of measurements vs observed streamflow data using $10 \mathrm{yr}$ of data from the Leaf River watershed. This plot was obtained by time differencing of the original streamflow data series. For more information, refer to Eqs. (4) and (5) in the text. The solid line represents a flexible spline function fit through the scattered data.

ure 4 shows a scatterplot of the observed streamflow data against error deviation of the measurements using $10 \mathrm{yr}$ of data from the Leaf River watershed. The solid line was fit through the scattered data using a flexible spline function, and this relationship was used when computing the likelihood function to specify the variance of the streamflow measurement error at each time step as a function of the observed streamflow level.

Successful implementation of the EnKF within SODA also depends strongly on a realistic estimate of the model error. The absolute and relative sizes of the model and measurement errors directly control ensemble spread among the ensemble members during the propagation and update steps, and therefore affect the mean ensemble model forecasts and associated prediction uncertainty ranges. There is usually little external information available to guide specification of the model error. Further, there can be little compelling information to guide specification of the characteristics and magnitude of the errors in the inputs to the model. In this work, instead of perturbing the input data (MAP and PET) using an assumed form for input error model (e.g., Evensen 1994; Moradkhani et al. 2005a,b), we treat input and model error jointly as a combined stochastic forcing term (hereafter simply called model error) and estimate its properties separately for each ensemble run within SODA. The most obvious advantage of this approach is that we can reliably estimate the size of this joint stochastic error term as a function of flow level. Specifically, we estimate the size of the model error as a function of flow level using total error decomposition, that is, by subtracting the measurement error function (described above) from the residual time 

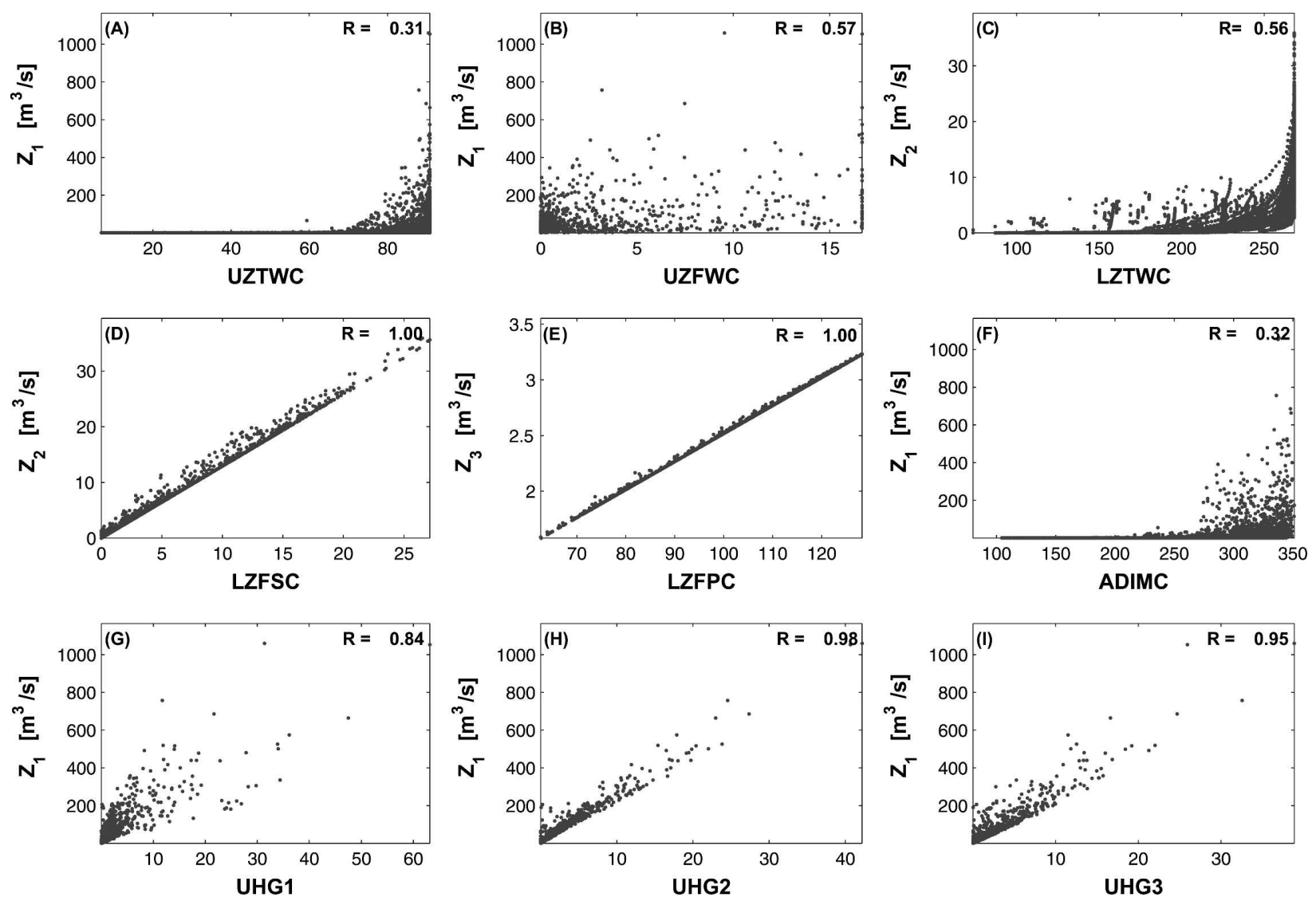

FIG. 5. Two-dimensional scatterplots of SAC-SMA simulated model states against their corresponding channel inflow components $Z_{1}, Z_{2}$, and $Z_{3}$, for the "most likely" SCEM-UA parameter set obtained for the calibration period.

series obtained each time the SAC-SMA model is evaluated against an observed time series of data. The model error variance is, therefore, both dependent on flow level and conditional (as it should be) on the specific parameter set used for each ensemble run.

Although our implementation of the SAC-SMA model contains nine state variables (six SAC-SMA soil storages and three $\mathrm{NC}$ routing storages) we determined that, because of the strongly nonlinear state-to-output transition equations, the EnKF linear updating rule could only be satisfactorily used for updating a subset of these. Figure 5 shows two-dimensional scatterplots of the nine SAC-SMA simulated model states against their corresponding channel inflow components $Z_{1}, Z_{2}$, and $Z_{3}$, using the SCEM-UA most likely parameter set obtained by calibration to data period WY 1953-60. Although we focus here on the calibration period only, similar scatterplots and correlation structures were obtained for the 28-yr evaluation period. Notice that only some of the plots indicate strong linear correlations. For the purposes of this study, we therefore decided to recursively update only the three model states UHG3,
LZFSC, and LZFPC, corresponding respectively to the three main components of channel flow (quick, intermediate, and slow responses). UHG3 is the content of the last of the sequential cascade of reservoirs used to rout channel component $Z_{1}$; LZFSC is the content of the lower-zone free secondary water soil store, which controls the early portion of the baseflow recession; and LZFPC is the content of the lower-zone free primary water soil store, which controls the late portion of the baseflow recession.

In addition, we used the following assumption to improve performance of the EnKF. The available data provide us with measurement information about the total channel streamflow rather than about the individual channel flow components $Z_{1}, Z_{2}$, and $Z_{3}$. However, both the conceptual structure of the SAC-SMA model and examination of the streamflow hydrograph reveal that each of these channel components tends to dominate the magnitude of channel streamflow at different periods of time $-Z_{1}$ (quick response) dominates during the rising and early recession stages of the storm event, $Z_{2}$ (intermediate response) dominates during the 


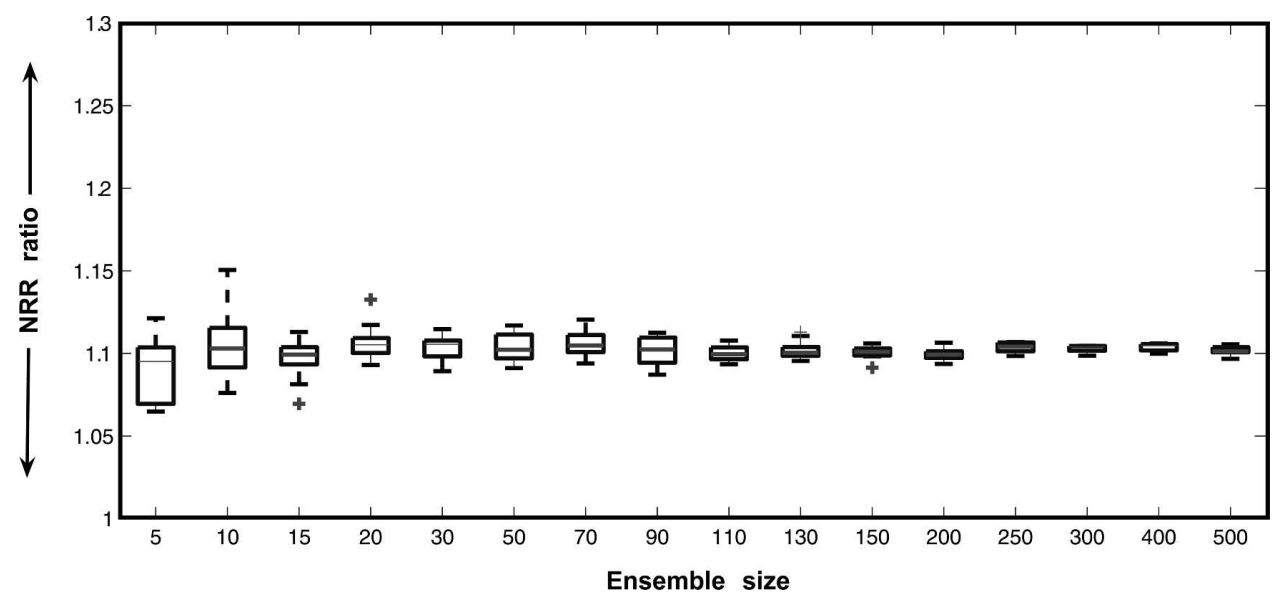

FIG. 6. NRR as a function of ensemble size for the "most likely" SODA parameter set derived for the calibration period.

later recession stages of the storm event, and $Z_{3}$ (slow response) dominates during the interstorm baseflow recession periods. We therefore assumed that the simulated (i.e., model computed) estimates $Z_{1}(\theta), Z_{2}(\theta)$, and $Z_{3}(\theta)$ provide reasonable estimates of the relative fractions of the total streamflow observation $\tilde{Z}$ coming from each of the three components of channel flow. Based on this assumption, the measurement operator was modified so that

$\tilde{Z}_{i}(\theta)=\frac{Z_{i}(\theta)}{Z_{1}(\theta)+Z_{2}(\theta)+Z_{3}(\theta)} \cdot \tilde{Z} \quad$ for each $\quad \mathrm{i}=\{1,2,3\}$,

which, in effect, partitions the scalar streamflow measurement $\tilde{Z}_{t}$ at each time step into a vector of three values $\tilde{Z}_{1}, \tilde{Z}_{2}$, and $\tilde{Z}_{3}$.

Finally, a number of experiments were conducted to evaluate sensitivity of filter and parameter estimation performance to the number of state-vector ensembles to be used in the EnKF implementation. Figure 6 shows a plot of the normalized rmse ratio (NRR) against EnKF ensemble size for the SODA estimated best parameter set for a given ensemble size. NRR is calculated in two steps: first the ratio of the time-averaged rmse of the ensemble mean to the mean rmse of the ensemble members is computed $(\mathrm{Ra})$, following which $\mathrm{Ra}$ is divided by $\sqrt{((N+1) / 2 N)}$, where $N$ denotes the ensemble size (see Fig. 2) (see Anderson 2001; Moradkhani et al. 2005a). A value of NRR close to 1.0 is desirable; NRR greater than 1.0 indicates that the ensemble has too little spread, whereas NRR $<1$ indicates too much spread. Figure 6 shows that the calibrated model gives an NRR value close to unity, indicating that the size of the ensemble is of the correct order of magnitude and that ensemble sizes larger than 50 do not provide improved results. An ensemble size of $N=50$ was used for all the studies reported in this paper. Note that NRR values nearly identical to unity were obtained when the model parameters were allowed to vary within the bounds obtained using a classical Bayesian SCEM-UA calibration.

\section{Discussion of results}

Results of the model calibration phase of this study are shown in Figs. 7 to 9 using WY 1953 for illustration. In all cases where a streamflow hydrograph is shown, box plot symbols are used to denote the median, lower, and upper quartile values of the confidence intervals for the streamflow data. Figure 7a shows the benchmark deterministic model case where the SAC-SMA model was calibrated in the conventional manner (which implicitly assumes an accurate model structure), without state-variable updating, using the method described in section 5 to specify variation of output measurement error variance with flow level and the SCEMUA algorithm to estimate parameter uncertainty. While the model-simulated estimates of streamflow look reasonable, the simulated $95 \%$ confidence intervals for streamflow (dark gray region; generated by projecting parameter uncertainty into the output space) are clearly too narrow and do not include the data, indicating that the estimation procedure is placing too much confidence in the validity of the model. Figure $7 \mathrm{~b}$ shows the stochastic model case where the SAC-SMA model was calibrated using SODA (with EnKF recursive state-variable updating applied to each parameter set). The simulated overall $95 \%$ confidence intervals for streamflow, representing both parameter uncer- 

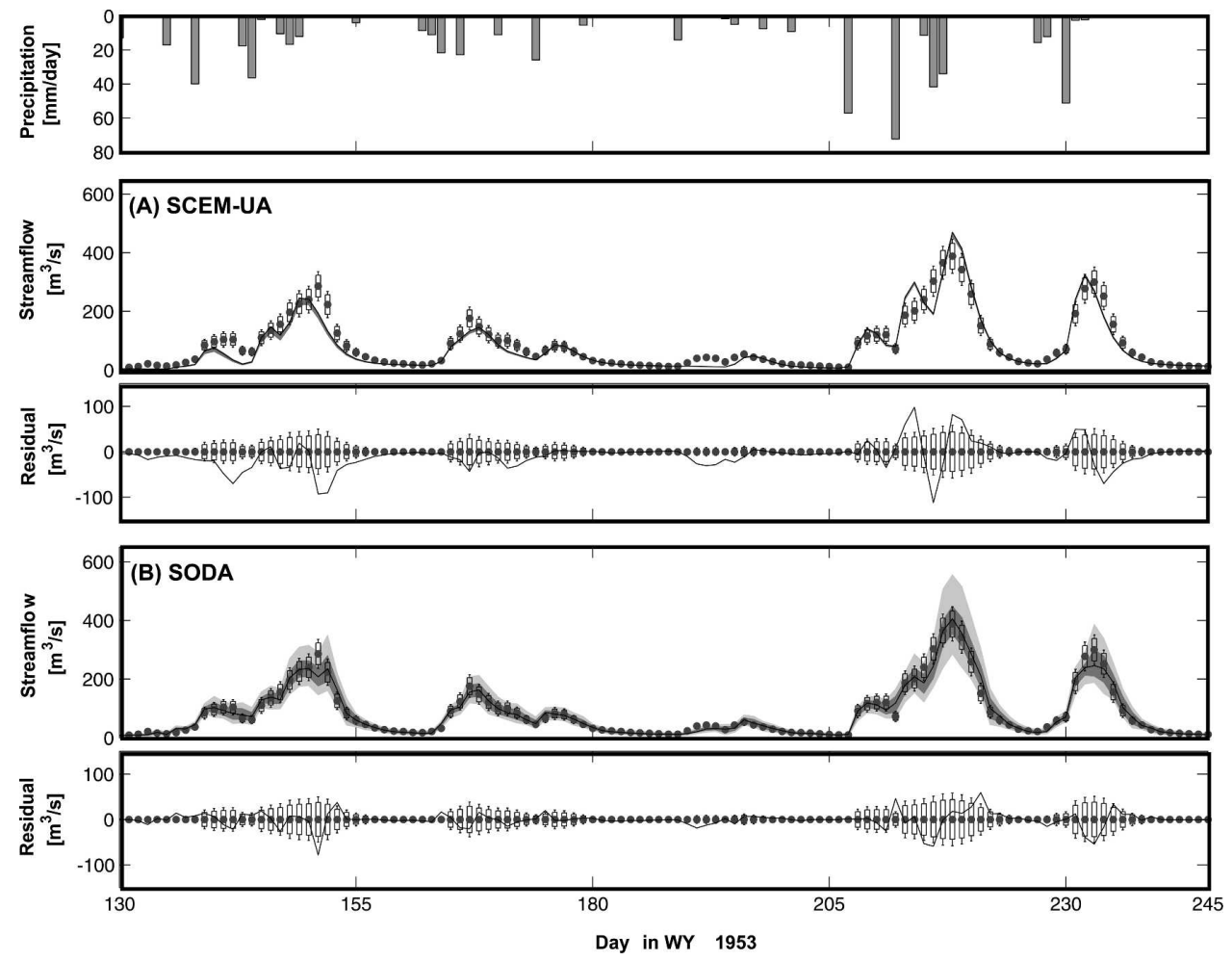

FIG. 7. Streamflow hydrograph predictions for calibration year WY 1953 for the (a) SCEM-UA deterministic model case parameters and the (b) SODA stochastic model case parameters. Streamflow data measurement uncertainty is indicated using box plots. In each case, the prediction given by the "most likely" parameter set is indicated using a solid black line, and the prediction uncertainty ranges corresponding to parameter uncertainty are indicated by the dark gray region. For SODA the additional uncertainty due to model error is indicated by the light gray region.

tainty (dark gray) and state-variable uncertainty caused by model structure and input errors (light gray) are now reasonable and tend to bracket the observations. Figure 8 and Table 1 report the associated posterior parameter uncertainties for these two cases. The parameter uncertainty ranges for the stochastic model case (shown to the right of each subplot) are much wider than for the deterministic model case. Also, the estimated parameter values are different, which has implications that will be discussed later. Figure 9 shows the mean innovations of the ensemble (dark line) and ensemble innovation spread (gray area) for the three updated model states, UHG3, LZFSC, and LZFPC, for the "most likely" parameter set identified using SODA. The relative corrections to UHG3 and LZFSC are seen to be similar, indicating inability of the filter to separate out information relating to these two faster flow components, while the corrections to LZFPC vary in strength at different times and indicate significant corrections to the state variable controlling the model estimates of slow response baseflow.

Results for the model evaluation phase of this study are shown in Fig. 10 using WY 1977 for illustration. Figure 10a shows the performance of the model for the benchmark deterministic model calibration case. The model forecasts tend to be poor and fall well outside the $95 \%$ streamflow measurement confidence intervals at times. In contrast the performance of the stochastic model seems good and the prediction uncertainties generally bracket the streamflow measurements.

Table 2 reports the variance of the model residuals computed for different flow ranges. In addition to the deterministic case (labeled SCEM-UA) and the stochastic case (labeled SODA Updating), the table includes results in which the stochastic case parameter estimates are used for deterministic streamflow prediction without online data assimilation (labeled SODA No updating). This table illustrates a number of important results. 1) Recursive state estimation using the EnKF applied to only three of the nine model states results in significantly better model forecasts during both the calibration period (52\% improvement) and evaluation period (34\% improvement). The model performs less well over the evaluation period, indicating 

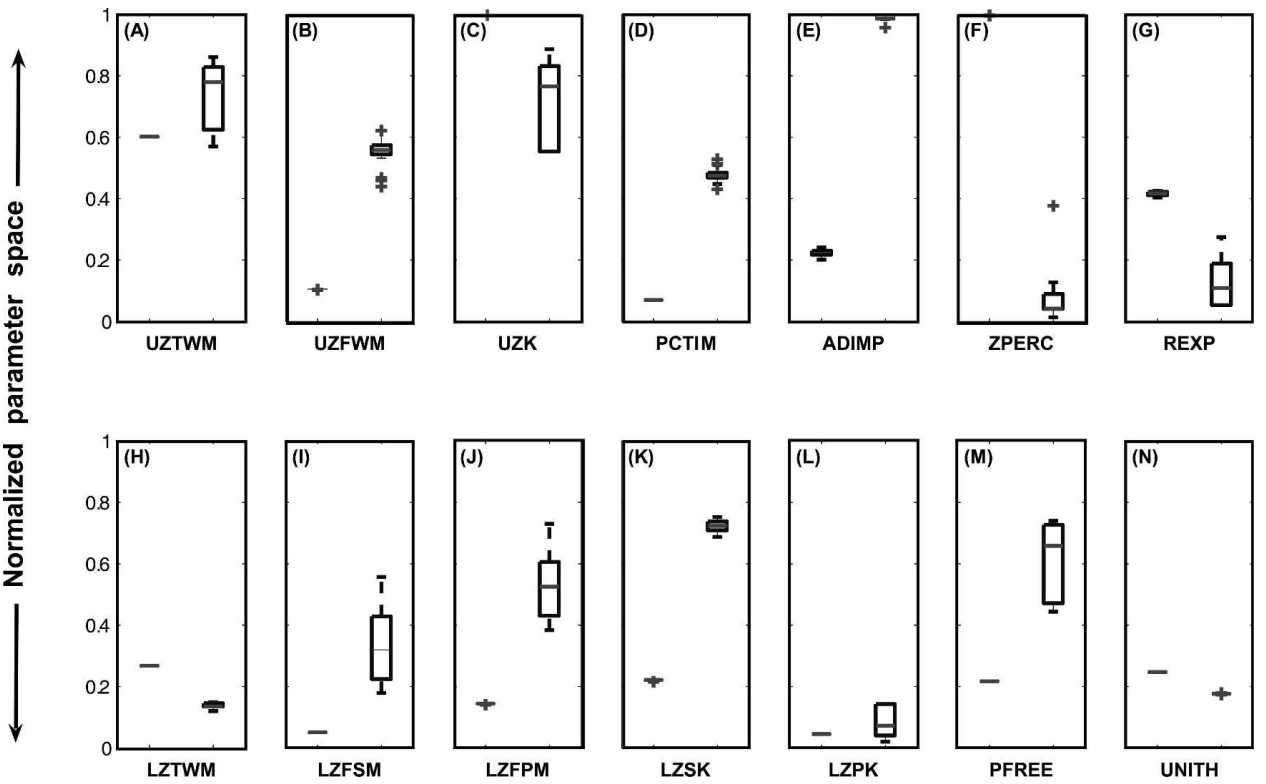

FIG. 8. Posterior uncertainty ranges (box plots) for the (left) SCEM-UA deterministic model case parameters and the (right) SODA stochastic model case parameters resulting from model calibration using the period WY 1953-60. The parameter uncertainties are scaled relative to their prior ranges to obtain normalized values.

that the statistical properties of the rainfall-soil moisture-runoff transformation may be variable in time [i.e., the batch calibration assumption of time-invariant model structure and parameters may be incorrect—see also Moradkhani et al. (2005a,b)]. 2) State estimation results in much larger relative improvements to model performance at lower flows ( $\sim 80 \%$ improvement) than at high flows $(\sim 40 \%$ improvement for calibration/
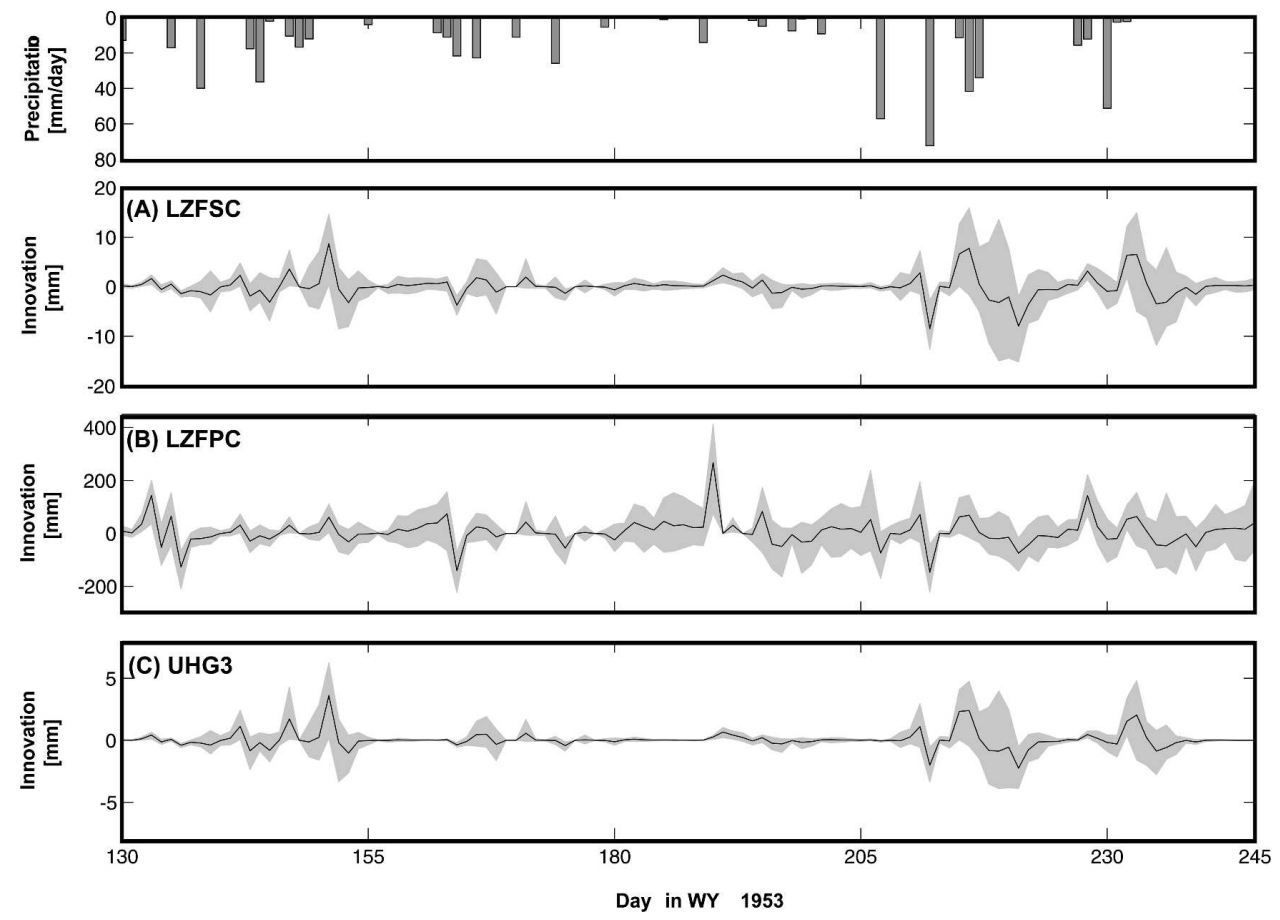

FIG. 9. Mean ensemble innovations (dark line) for the LZFSC, LZFPC, and UHG3 states in the SAC-SMA model for calibration year WY 1953. Results correspond to the mode of the posterior distribution identified using SODA. Dark gray ranges reflect uncertainty in the ensemble members. 

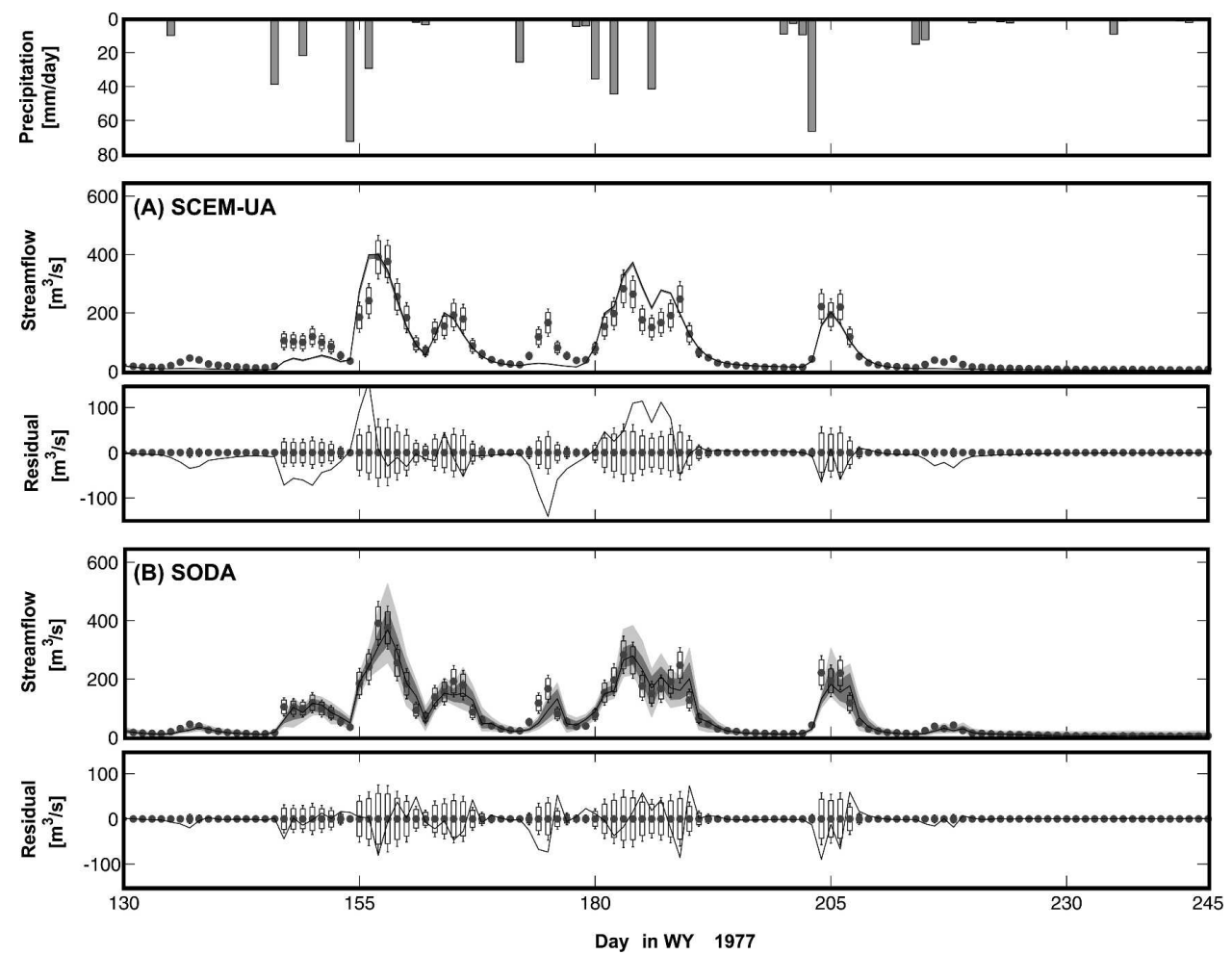

FIG. 10. Streamflow hydrograph predictions for evaluation year WY 1977 for the (a) SCEM-UA deterministic model case parameters and the (b) SODA stochastic model case parameters. Streamflow data measurement uncertainty is indicated using box plots. In each case, the prediction given by the "most likely" parameter set is indicated using a solid black line, and the prediction uncertainty ranges corresponding to parameter uncertainty are indicated by the dark gray region. For SODA the additional uncertainty due to model error is indicated by the light gray region.

$\sim 30 \%$ improvement for evaluation). This of course correctly reflects the fact that the error variance of the streamflow measurement data increases with streamflow level. 3) The "most likely" parameter set estimated using SODA gives significantly better forecast performance than the one estimated using SCEM-UA for both the calibration and evaluation periods (SODA No updating case). This confirms our view (see Vrugt et al. 2005) that proper acknowledgment of state-variable uncertainty (in addition to parameter uncertainty and output measurement error) can result in better estimates for the parameters (in the sense that they are less corrupted by system and other kinds of errors). Further support for this claim is given in Clark and Vrugt (2006).

Figure 11 illustrates another test of model perfor-

TABLE 2. Summary statistic (rmse) for the one-day-ahead streamflow forecasts over the calibration and evaluation period using the SCEM-UA and SODA methods with and without recursive state updating. The error statistic is computed for different flow groups.

\begin{tabular}{|c|c|c|c|c|c|c|}
\hline \multirow[b]{3}{*}{ Flow group $\left(\mathrm{m}^{3} \mathrm{~s}^{-1}\right)$} & \multicolumn{3}{|c|}{ Calibration (WY 1953-60) } & \multicolumn{3}{|c|}{ Evaluation (WY 1961-88) } \\
\hline & \multirow[b]{2}{*}{ SCEM-UA } & \multicolumn{2}{|c|}{ SODA } & \multirow[b]{2}{*}{ SCEM-UA } & \multicolumn{2}{|c|}{ SODA } \\
\hline & & No updating & Updating & & No updating & Updating \\
\hline $0-5$ & 3.36 & 1.73 & 0.55 & 3.42 & 1.44 & 0.65 \\
\hline $5-10$ & 8.22 & 3.91 & 1.76 & 7.02 & 3.36 & 1.71 \\
\hline $10-25$ & 13.18 & 8.59 & 4.15 & 10.83 & 8.18 & 5.68 \\
\hline $25-50$ & 19.74 & 15.54 & 9.19 & 22.78 & 15.67 & 10.99 \\
\hline $50-100$ & 28.41 & 25.95 & 17.90 & 32.42 & 28.52 & 17.41 \\
\hline $100-250$ & 69.89 & 58.29 & 33.03 & 53.83 & 53.06 & 43.12 \\
\hline$>250$ & 129.81 & 121.41 & 78.47 & 128.63 & 111.45 & 102.29 \\
\hline Overall & 22.47 & 18.31 & 11.07 & 27.83 & 24.67 & 18.28 \\
\hline
\end{tabular}



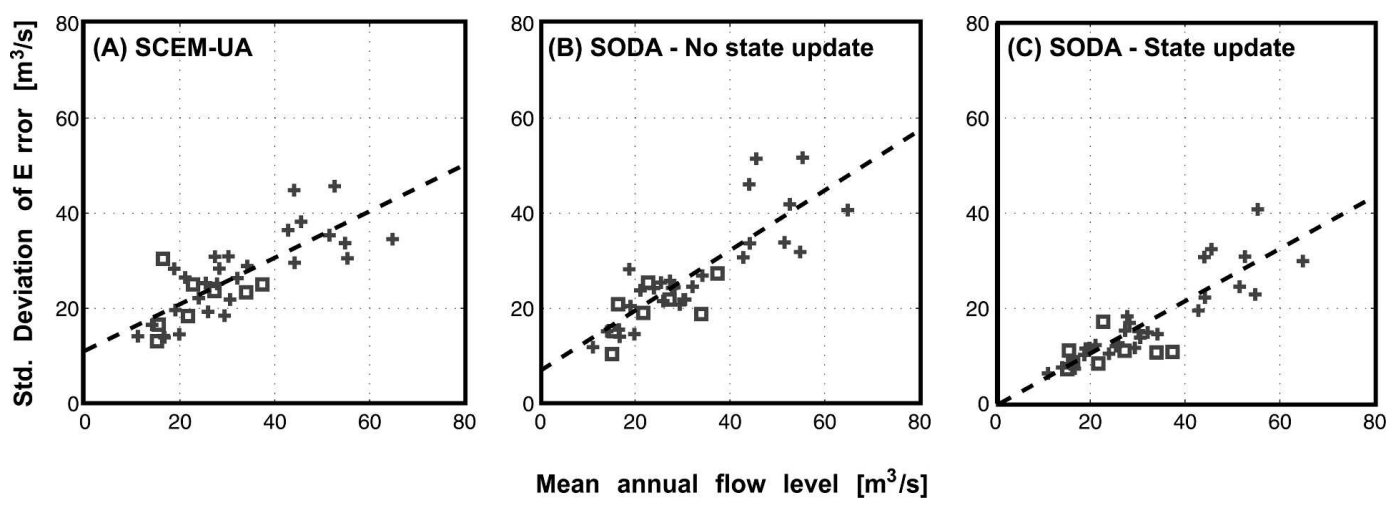

FIG. 11. Annual mean standard deviation of one-day-ahead streamflow forecast errors as function of mean annual flow level for the (a) SCEM-UA deterministic model case parameters, and the (b) SODA stochastic model case parameters without state updating and (c) with state updating. Calibration years are plotted using the box symbol, and evaluation years are plotted using the "+" symbol.

mance and consistency. For each of the 36 water years used in this study ( 8 calibration plus 28 evaluation), the annual standard deviation of the one-day-ahead streamflow forecast errors is plotted against the annual mean flow level for that year. Each box symbol indicates a calibration year and each "+" symbol indicates an evaluation year. In all three cases the symbols appear to fall approximately on a straight line indicating that calibration and evaluation period performance are mutually consistent. The slopes of the lines indicate that forecast errors tend to be larger for wetter years (as reported also by Hsu et al. 2002). However, in the case of SODA, the points are less scattered, the slope of the regression line is smaller and the intercept approximately intersects the origin, indicating better and more consistent forecast performance. Further, a statistical check of the autocorrelation functions of the residuals showed that for both of the cases without state updating the forecast errors are strongly correlated. In contrast, the SODA forecast errors are essentially white (uncorrelated), indicating that most of the bias in the one-dayahead streamflow predictions is removed by the recursive state updating procedure.

Finally, it is worth mentioning that the performance of the SAC-SMA model with recursive state estimation is very similar to that obtained for the same watershed by Hsu et al. (2002) using the deterministic SOLO artificial neural network (ANN) approach having 1350 parameters. The ANN results provide an indication of the limits to achievable forecasting performance for this watershed when using rainfall to predict streamflow. These results indicate that the SAC-SMA model is a relatively good representation of the rainfall-soil moisture-runoff transformation process for medium-sized humid watersheds such as the Leaf River. Taken together with the parameter uncertainty results reported in Fig. 8 and Table 1, they also further support our view that [contrary to suggestions by Jakeman and Hornberger (1993)] the rainfall-runoff data can and do contain sufficient information for identifying models of this level of complexity $(14+$ parameters and 9 state variables).

\section{Summary and conclusions}

While conceptual watershed models are widely used for operational streamflow forecasting their strongly nonlinear structural equations (including thresholdtype discontinuities) have historically posed a challenge to the application of computer-based systems' methods for automated model identification, and parameter and state estimation. Progress in stochastic global optimization methods has helped to diminish the difficulties associated with parameter estimation. The recent development of ensemble-based techniques and filtering strategies for sequential data assimilation now make it possible (in principle) to implement techniques that properly account for data (input and output), state variable, parameter, and model structural uncertainties, although the details of how such implementation can be achieved are still an active area of research.

This paper has demonstrated the applicability of the SODA method for joint parameter-state estimation and operational ensemble streamflow forecasting using the SAC-SMA conceptual watershed model. SODA merges the strengths of the ensemble Kalman filter for recursive data assimilation to update model states, and the shuffled complex evolution metropolis algorithm for batch data assimilation to estimate time-invariant values for the model parameters. The algorithm was implemented using a LAM/MPI distributed processing interface and Octave programming environment to 
maximize computing efficiency, using 25 Pentium IV $3.40-\mathrm{GHz}$ processing nodes. Each stochastic joint calibration and ensemble state estimation run reported in this paper using 50000 SCEM-UA generated parameter combinations required approximately $22 \mathrm{~h}$ of CPU time.

The performance of the stochastic SODA method (using an ensemble size of 50) was compared to a conventional deterministic model calibration approach. The heteroscedastic error variance properties of the streamflow measurement error were estimated from the data using a nonparametric approach and the heteroscedastic error variance properties of the model structural error were estimated (for each parameter set) from the model residuals by subtraction. Because the EnKF uses a linear rule for state updating we decided to update only the three of the nine model states that showed strong cross covariances with the three main components of channel flow (quick, intermediate, and slow responses). A simple but reasonable assumption was used to partition the scalar streamflow measurement at each time step into a vector corresponding to the three flow component values. The results may be summarized as follows:

1) The conventional deterministic approach gives narrow estimates of streamflow uncertainty that do not include the data, indicating that the deterministic estimation procedure places too much confidence in the validity of the model. In contrast, the stochastic SODA approach gives reasonable streamflow confidence intervals (neither too wide nor too narrow) that bracket the observations.

2) The parameter uncertainty ranges for the stochastic model case (SODA) are wider than for the deterministic model case (SCEM-UA) and lie in different areas of the feasible parameter space. Further, the SODA "most likely" parameter set gives significantly better forecast performance (in nonupdating/ simulation mode) than its SCEM-UA counterpart, indicating that proper treatment of state variable, parameter, and output measurement uncertainties can result in better (i.e., less biased) parameter estimates. This may increase the prospects for finding useful regionalization relationships.

3) Although data assimilation via recursive state estimation was applied to only three of the nine model states, significant improvements in SAC-SMA model forecast performance were realized (on the order of $30 \%$ to $50 \%$ ).

4) State estimation resulted in larger relative improvements to model performance at lower flows than at higher flows, reflecting the fact that streamflow measurement error variance increases with streamflow level. Further, SODA forecast errors were essentially white (uncorrelated), indicating that most of the bias in the one-day-ahead streamflow predictions was removed by recursive state updating.

5) The calibrated SAC-SMA model with stochastic state-variable uncertainty propagation and updating provides streamflow forecast estimates that approach the accuracy achievable using a sophisticated 1350-parameter artificial neural network approach. These results suggest that the 16-parameter SACSMA model is a relatively good representation of the rainfall-soil moisture-runoff transformation process for medium-sized humid watersheds such as the Leaf River.

On the negative side, we also found that the calibrated model performs less well over the evaluation period, suggesting that the statistical properties of the rainfall-soil moisture-runoff transformation may be time varying in a manner not adequately captured by the SODA procedure. Part of this might be due to the fact that the evaluation period showed on average considerable higher flow values. In particular, the SCEMUA batch calibration approach assumes that the system structure (as reflected in the structural equations and parameters) is time invariant and that the accuracy of the model (as reflected in the temporal trajectory of model error bias) is stationary. Further, our approach does not yet properly account for input errors, particularly of the nonstationary kind arising from nondetection of precipitation (caused by inadequate gauge density and other factors). Nevertheless, the results of this study indicate that the SODA method has the potential for ready implementation into operational forecasting procedures, and the capability of providing improved ensemble streamflow forecasts without requiring significant modifications to operational model codes or software. Research aimed at further improvements in algorithm applicability, performance, and efficiency are ongoing and will be reported in due course. As always, we invite dialogue with others interested in these topics. Software used in this and related work can be found online at www.science.uva.nl/ibed/cbpg/products and/ or at www.sahra.arizona.edu/software.

Acknowledgments. The first author is supported by the LANL Director's Funded Postdoctoral program. In addition, we are grateful for partial support received from the National Weather Service (Grants NA87WHO582 and NA07WH0144), the SAHRA Center for Sustainability of Semi arid Hydrology and Riparian Areas at the University of Arizona (NSF Grant 
EAR-9876800), and for computer support provided by the SARA center for parallel computing at the University of Amsterdam.

\section{REFERENCES}

Anderson, H., 2001: An ensemble adjustment filter for data assimilation. Mon. Wea. Rev., 129, 2884-2903.

Arulampalam, M. S., S. Maskell, N. Gordon, and T. Clapp, 2002: A tutorial on particle filters for online nonlinear/nonGaussian Bayesian tracking. IEEE Trans. Signal Process., 50 (2), 174-188.

Awwad, H. M., and J. B. Valdés, 1992: Adaptive parameter estimation for multi-site hydrologic forecasting. J. Hydraul. Eng., 118, 1201-1221.

—,$~ —$, and P. J. Restrepo, 1994: Streamflow forecasting for Han River basin, Korea. J. Water Resour. Plann. Manage., 120, 651-673.

Beven, K. J., and A. M. Binley, 1992: The future of distributed models: Model calibration and predictive uncertainty. $\mathrm{Hy}$ drol. Processes, 6, 279-298.

Box, G. E. P., and G. C. Tiao, 1973: Bayesian Inference in Statistical Analyses. Addison-Wesley-Longman, 585 pp.

Boyle, D. P., H. V. Gupta, and S. Sorooshian, 2000: Towards improved calibration of hydrologic models: Combining the strengths of manual and automatic methods. Water Resour. Res., 36, 3663-3674.

,-- , and -2001 : Towards improved streamflow forecasts: The value of semi-distributed modeling. Water Resour. Res., 37, 2749-2759.

Bras, R. L., and P. Restrepo-Posada, 1980: Real time automatic parameter calibration in conceptual runoff forecasting models. Proc. Third Int. Symp. on Stochastic Hydraulics, 61-70.

- , and I. Rodriguez-Iturbe, 1985: Random Functions and Hydrology. Addison-Wesley, $545 \mathrm{pp}$.

Brazil, L. E., and M. D. Hudlow, 1981: Calibration procedures used with the National Weather Service Forecast System. Water and Related Land Resources, Y. Y. Haimes and J. Kindler, Eds., Pergamon Press, 457-466.

Burnash, R. J. C., R. L. Ferral, and R. A. McGuire, 1973: A generalized streamflow simulation system: Conceptual models for digital computers. National Weather Service, NOAA, and the State of California Department of Water Resources Tech. Rep., Joint Federal-State River Forecast Center, Sacramento, CA, 68 pp.

Cañizares, R., H. Madsen, H. R. Jensen, and H. J. Vested, 2001: Developments in operational shelf sea modeling in Danish waters. Estuarine Coastal Shelf Sci., 53, 595-605.

Clark, M. P., and J. A. Vrugt, 2006: Unraveling uncertainties in hydrologic model calibration: Addressing the problem of compensatory parameters. Geophys. Res. Lett., 33, L06406, doi:10.1029/2005GL025604.

Dette, H., A. Munk, and T. Wagner, 1998: Estimating the variance in nonparametric regression-What is a reasonable choice? $\mathrm{J}$. Roy. Stat. Soc., 60B, 751-764.

Duan, Q., V. K. Gupta, and S. Sorooshian, 1992: Effective and efficient global optimization for conceptual rainfall-runoff models. Water Resour. Res., 28, 1015-1031.

_, S. Sorooshian, and V. K. Gupta, 1994: Optimal use of the SCE-UA global optimization method for calibrating watershed models. J. Hydrol., 158, 265-284.

Eaton, J. W., 1998: Octave Version 1. Department of Chemical
Engineering, University of Wisconsin. [Available online at http://www.octave.org.]

_- 2001: Octave: Past, Present and Future. Proc. Second Int Workshop on Distributed Statistical Computing, Vienna, Austria, Technical University of Vienna, 1-17.

Evensen, G., 1992: Using the extended Kalman filter with a multilayer quasi-geostrophic ocean model. J. Geophys. Res., 97 (C11), 17 905-17 924.

_ 1994: Sequential data assimilation with a nonlinear quasigeostrophic model using Monte Carlo methods to forecast error statistics. J. Geophys. Res., 99, $10143-10162$.

Fernández, J., A. Cañas, A. F. Díaz, J. González, J. Ortega, and A. Prieto, 2003: Performance of message-passing MATLAB toolboxes. Lect. Notes Comput. Sci., 2565, 228-241.

—, M. Anguita, S. Mota, A. Cañas, E. Ortigosa, and F. J. Rojas, 2004: MPI toolbox for Octave. Proc. Sixth Int. Conf. on High Performance Computing for Computational Science, Valencia, Spain, Polytechnic University of Valencia, 1-6.

Freer, J., K. J. Beven, and B. Ambroise, 1996: Bayesian estimation of uncertainty in runoff prediction and the value of data: An application of the GLUE approach. Water Resour. Res., 32, 2161-2173.

Georgakakos, H., and J. A. Sperflage, 1995: Hydrologic forecast system-HFS: A user's manual. HRC Tech. Note 1, Hydrologic Research Center, San Diego, CA, 17 pp.

_, H. Rajaram, and S. G. Li, 1988: On improved operational hydrologic forecasting of streamflows. Iowa Institute of Hydraulic Research Rep. 325, 162 pp.

Gordon, N. D., D. Salmond, and A. F. M. Smith, 1993: Novel approach to nonlinear and non-Gaussian Bayesian state estimation. Proc. Inst. Electr. Eng., Part F, 140, 107-113.

Gupta, H. V., S. Sorooshian, and P. O. Yapo, 1998: Towards improved calibration of hydrologic models: Multiple and noncommensurable measures of information. Water Resour. Res., 34, 751-763.

,-- T T. S. Hogue, and D. P. Boyle, 2003: Advances in automatic calibration of watershed models. Calibration of Watershed Models, Q. Duan et al., Eds., Water Science Applications Series, Vol. 6, Amer. Geophys. Union, 113-124.

Gupta, V. K., and S. Sorooshian, 1985: The relationship between data and the precision of estimated parameters. J. Hydrol., 81, 57-77.

Hall, P., J. W. Kay, and D. M. Titterington, 1990: Asymptotically optimal difference-based estimation of variance in nonparametric regression. Biometrika, 77, 521-528.

Hogue, T. S., S. Sorooshian, H. V. Gupta, A. Holz, and D. Braatz, 2000: A multi-step automatic calibration scheme (MACS) for river forecasting models. J. Hydrometeor., 1, 524-542.

— , H. V. Gupta, and S. Sorooshian, 2005: A "user-friendly" approach to parameter estimation in hydrologic models. $J$. Hydrol., 320, 202-217.

Hsu, K., H. V. Gupta, X. Gao, S. Sorooshian, and B. Imam, 2002: Self-organizing linear output map (SOLO): An artificial neural network suitable for hydrologic modeling and analysis. Water Resour. Res., 38, 1302, doi:10.1029/2001WR000795.

Jakeman, A., and G. M. Hornberger, 1993: How much complexity is warranted in a rainfall-runoff model? Water Resour. Res., 29, 2637-2649.

Kalman, R., 1960: New approach to linear filtering and prediction problems. J. Basic Eng., 82D, 35-45.

Kavetski, D., S. W. Franks, and G. Kuczera, 2003: Confronting input uncertainty in environmental modeling. Calibration of 
Watershed Models, Q. Duan et al., Eds., Water Science Applications Series, Vol. 6, Amer. Geophys. Union, 49-68.

Keesman, K. J., 1990: Set theoretic parameter estimation using random scanning and principal component analysis. Math. Comput. Simul., 32, 535-543.

Kitanidis, P. K., and R. L. Bras, 1980a: Real time forecasting with a conceptual hydrologic model, 1, Analysis of uncertainty. Water Resour. Res., 16, 1025-1033.

— , and _ 1980b: Real time forecasting with a conceptual hydrologic model, 2, Applications and results. Water Resour. Res., 16, 1034-1044.

Klepper, O., H. Scholten, and J. P. G. van de Kamer, 1991: Prediction uncertainty in an ecological model of the Oosterschelde Estuary. J. Forecasting, 10, 191-209.

Kuczera, G., 1983a: Improved parameter inference in catchment models: 1. Evaluating parameter uncertainty. Water Resour. Res., 19, 1151-1162.

_ 1983 b: Improved parameter inference in catchment models: 2. Combining different kinds of hydrologic data and testing their compatibility. Water Resour. Res., 19, 1163-1172.

_- 1997: Efficient subspace probabilistic parameter optimization for catchment models. Water Resour. Res., 33, 177-185.

Madsen, H., 2000: Automatic calibration of a conceptual rainfallrunoff model using multiple objectives. J. Hydrol., 235, 276288.

— , and C. Skotner, 2005: Adaptive state-updating in real-time river flow forecasting-A combined filtering and error forecasting procedure. J. Hydrol., 308, 302-312.

Miller, R. N., M. Ghil, and F. Ghautiez, 1994: Advanced data assimilation in strongly nonlinear dynamical systems. $J$. Atmos. Sci., 51, 1037-1055.

Misirli, F., 2003: Improving efficiency and effectiveness of Bayesian recursive parameter estimation for hydrologic models. Ph.D. dissertation, The University of Arizona.

Moradkhani, H., S. Sorooshian, H. V. Gupta, and P. R. Hauser, 2005a: Dual state-parameter estimation of hydrological models using ensemble Kalman filter. Adv. Water Res., 28, 135147.

— K. Ksu, H. V. Gupta, and S. Sorooshian, 2005b: Uncertainty assessment of hydrologic model states and parameters: Sequential data assimilation using the particle filter. Water Resour. Res., 41, W05012, doi:10.1029/2004WR003604.

Refsgaard, J. C., 1998: Validation and intercomparison of different updating procedures for real-time forecasting. Nord. Hydrol., 28, 65-84.

Reichle, R. H., D. Entekhabi, and D. B. McLaughlin, 2001: Downscaling radio brightness measurements for soil moisture estimation: A four dimensional variational data assimilation approach. Water Resour. Res., 37, 2353-2364.

Rice, J., 1984: Bandwidth choice for nonparametric kernel regression. Ann. Stat., 12, 1215-1230.

Seifert, B., T. Gasser, and A. Wolf, 1993: Nonparametric estimation of residual variance revisited. Biometrika, 80, 373-383.

Seo, D. J., V. Koren, and N. Cajina, 2003: Real-time variational assimilation of hydrologic and hydrometeorological data into operational hydrologic forecasting. J. Hydrometeor., 4, 627641.

Sorooshian, S., and J. A. Dracup, 1980: Stochastic parameter estimation procedures for hydrologic rainfall-runoff models: Correlated and heteroscedastic error cases. Water Resour. Res., 16, 430-442.
- and V. K. Gupta, 1983: Automatic calibration of conceptual rainfall-runoff models: The question of parameter observability and uniqueness. Water Resour. Res., 19, 260-268.

—, Q. Duan, and V. K. Gupta, 1993: Calibration of rainfallrunoff models: Application of global optimization to the Sacramento soil moisture accounting model. Water Resour. Res., 29, 1185-1194.

Thiemann, M., M. W. Trosset, H. V. Gupta, and S. Sorooshian, 2001: Bayesian recursive parameter estimation for hydrologic models. Water Resour. Res., 37, 2521-2535.

Todini, E., A. Szollosi-Nagy, and E. F. Wood, 1976: Adaptive state-parameter estimation algorithm for real time hydrologic forecasting: A case study. Proc. IIASA/WMO Workshop on the Recent Developments in Real Time Forecasting/Control of Water Resources Systems, Laxenburg, Austria, IIASALaxenburg.

van Straten, G., and K. J. Keesman, 1991: Uncertainty propagation and speculation in projective forecasts of environmental change: A lake eutrophication example. J. Forecasting, 10, 163-190.

Vrugt, J. A., W. Bouten, H. V. Gupta, and S. Sorooshian, 2002: Toward improved identifiability of hydrologic model parameters: The information content of experimental data. Water Resour. Res., 38, 1312, doi:10.1029/2001WR001118.

- , H. V. Gupta, L. Bastidas, W. Bouten, and S. Sorooshian, 2003a: Effective and efficient algorithm for multiobjective optimization of hydrologic models. Water Resour. Res., 39, 1214, doi:10.1029/2002WR001746.

$\_,-, W$. Bouten, and S. Sorooshian, 2003b: A shuffled complex evolution metropolis algorithm for optimization and uncertainty assessment of hydrologic model parameters. Water Resour. Res., 39, 1201, doi:10.1029/2002WR001642.

- C. G. H. Diks, H. V. Gupta, W. Bouten, and J. M. Verstraten, 2005: Improved treatment of uncertainty in hydrologic modeling: Combining the strengths of global optimization and data assimilation. Water Resour. Res., 41, W01017, doi:10.1029/2004WR003059.

_ , H. V. Gupta, S. C. Dekker, S. Sorooshian, T. Wagener, and W. Bouten, 2006a: Application of stochastic parameter optimization to the Sacramento Soil Moisture Accounting model. J. Hydrol., in press.

— B. Ó. Nualláin, B. A. Robinson, W. Bouten, S. C. Dekker, and P. M. A. Sloot, 2006b: Application of parallel computing to stochastic parameter estimation in environmental models. Comput. Geosci., in press.

Wagener, T., N. McIntyre, M. J. Lees, H. S. Wheater, and H. V. Gupta, 2003: Towards reduced uncertainty in conceptual rainfall-runoff modeling: Dynamic identifiability analysis. Hydrol. Processes, 17, 455-476.

Wood, E. F., and P. E. O'Connell, 1985: Real-time forecasting. Hydrological Forecasting, M. G. Anderson and T. P. Burt, Eds., John Wiley and Sons.

Yapo, P., H. V. Gupta, and S. Sorooshian, 1996: Automatic calibration of conceptual rainfall-runoff models: Sensitivity to calibration data. J. Hydrol., 181, 23-48.

Young, P. C., 2001: Data-based mechanistic modeling and validation of rainfall-flow processes. Model Validation, M. G. Anderson and P. G. Bates, Eds., John Wiley and Sons, 117161.

_ 2002: Advances in real-time flood forecasting. Philos. Trans. Roy. Soc. London, 360, 1433-1450. 